\title{
Efectos de la suplementación con L-carnitina en el síndrome de ascitis en los pollos de engorde criados a gran altura
}

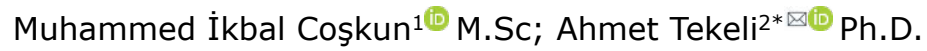

\begin{abstract}
${ }^{1}$ Van Yuzuncu Yıl University, Deparment of Animal Science, Van, Turkey.
2 Eskişehir Osmangazi University, Deparment of Animal Science, Eskişehir, Turkey.

*Corresponding: atekelim@gmail.com; atekeli@ogu.edu.tr
\end{abstract}

Recibido: Mayo 2018; Aceptado: Octubre 2018; Publicado: Febrero 2019.

\section{RESUMEN}

Objetivo. Investigar los efectos de la L-carnitina como un medio potencial para reducir la incidencia de ascitis en pollos de engorde y su relación con parámetros fisiológicos y bioquímicos. Material y métodos. Se utilizaron 300 pollos de engorde machos de un día de edad (Ross 308) en el ensayo. El grupo sin suplementación de L-carnitina (0) se asignó como control y los grupos que recibieron suplementos de 100, 150, 200 y 250 mg/L de L-carnitina en agua se asignaron como grupos de tratamiento. La prueba se completó en 35 días. Resultados. La suplementación de L-carnitina no tuvo ningún efecto significativo sobre el aumento de peso vivo, consumo de alimento, consumo de agua y tasa de conversión alimenticia. Los niveles de plasma sanguíneo y los parámetros del hemograma HDL, triglicéridos, CK RBC y $\mathrm{MCH}$ se vieron afectados significativamente por L-carnitina $(\mathrm{p}<0.05)$. El valor del $\mathrm{pH}$ del parámetro del gas en sangre se vio significativamente afectado por la suplementación con L-carnitina en los pollos de engorde con ascitis. El valor del $\mathrm{pH}$ del gas en la sangre aumentó significativamente con la suplementación de $100 \mathrm{mg} / \mathrm{L}$ de L-carnitina en comparación con la del control $(\mathrm{p}<0.05)$. Mientras que el $\mathrm{pH}$ de la sangre fue de 7.21 en los animales con ascitis, se determinó como 7.48 en animales sanos. Las concentraciones de $\mathrm{SO}_{2}$ y ctO $\mathrm{ct}_{2}$ fueron mayores en animales sanos, mientras que las concentraciones de $\mathrm{ctCO}_{2} \mathrm{P}$ y hemoglobina fueron mayores en animales ascíticos $(p<0.05)$. Conclusiones. Las tasas de mortalidad por ascitis a partir del grupo control se calcularon respectivamente como \%; 20.00, 18.33, 26.67 y $28.33 .76 .71 \%$ de las muertes totales de ascitis fueron en la quinta semana. Se concluyó que dosis bajas de suplementos de L-carnitina pueden tener efectos positivos en los pollos de engorde criados a gran altitude.

Palabras clave: L-carnitina, gas en sangre, hipertensión pulmonar, parámertos de rendimento, pollos de engorde (Fuente: USDA).

\begin{abstract}
Objective. Investigate the effects of L-carnitine as a potential means of reducing the incidence of ascites in broilers and its relationship with physiological and biochemical paramaters. Material and methods. One-day-old 300 male broiler chicks (Ross 308 ) were used in the trial. The group without $L$-carnitine supplementation (0) was assigned as control and the groups that received 100,150, 200 and $250 \mathrm{mg} / \mathrm{L} L$-carnitine supplementation in water were assigned as treatment groups. The trial was completed in 35 days. Results. $L$-carnitine supplementation did not have any significant effect on live weight gain, feed consumption, water consumption and feed conversion ratio. Levels of blood plasma and hemogram parameters HDL, Triglyceride, CK, RBC and MCH were significantly affected by L-carnitine $(p<0.05)$. Blood gas parameter $\mathrm{pH}$ value was significantly affected by $L$-carnitine supplementation in the broilers with ascites. Blood gas $\mathrm{pH}$ value significantly increased with $100 \mathrm{mg} / \mathrm{L} L$-carnitine supplementation compared to that of control $(\mathrm{p}<0.05)$. While blood $\mathrm{pH}$ was 7.21 in the animals with ascites, it was determined as 7.48 in healthy animals. Concentrations of $\mathrm{SO}_{2}$ and $\mathrm{ctO}_{2}$ were higher in healthy animals, while ctCO2P and hemoglobin concentrations were higher in ascitic animals $(p<0.05)$. Conclusions. Ascites mortality rates starting from the control group were calculated respectively as $\% ; 20.00,18.33,26.67,28.33$ and $28.33 \%$. $76.71 \%$ of total ascites deaths were in the 5th week. It was concluded that low doses of L-carnitine supplementation may have positive effects in the broilers grown at high altitude.
\end{abstract}

Keywords: Blood gas, broiler, L-carnitine, performance parameters, pulmonary hypertension (Source: USDA).

Como citar (Vancouver)

Coşkun MI, Tekeli A. Efectos de la suplementación con L-carnitina en el síndrome de ascitis (hipertensión pulmonar) en los pollos de engorde cultivados a gran altura. Rev MVZ Cordoba. 2019; 24(1):7127-7136. DOI: https://doi.org/10.21897/rmvz.1523

(C)EI (los) autor (es), Revista MVZ Córdoba 2018. Este artículo se distribuye bajo los términos de la licencia internacional Creative Commons Attribution 4.0 (https://creativecommons.org/licenses/by-sa/4.0/), que permite el uso sin restricciones, la distribución y la reproducción en cualquier medio, siempre que se otorgue el crédito apropiado al autor o autores originales y la fuente. 


\section{INTRODUCCIÓN}

La carnitina está químicamente en forma de $D$ y $L$ y sólo existe en la naturaleza y es importante para la nutrición humana y animal ( $\underline{1})$. La L-carnitina es un polvo blanco, altamente soluble en agua, caracterizado por una alta estabilidad térmica (hasta $200^{\circ} \mathrm{C}$ ). Su nombre químico es $\beta$-hydroxy- $Y$-trimetil-amino butirato. La L-carnitina también se conoce como vitamina BT (2) . La L carnitina tiene un efecto importante en la producción de energía y el metabolismo de la grasa en el cuerpo al proporcionar transporte mitocondrial de ácidos grasos de cadena larga y eliminación mitocondrial de ácidos grasos de cadena corta y media ( 3 ). Además de su uso en la alimentación animal (4), la L-carnitina también se ha utilizado en varios sectores de la salud, como la prevención de ataques cardíacos y otras afecciones cardíacas ( $\underline{5})$, la hemodiálisis (ㅁ) y el tratamiento de la infertilidad (ㅁ) .

La ascitis (hipertensión pulmonar) es un síndrome multifactorial resultante de las interacciones entre factores genéticos, ambientales, dietéticos y fisiológicos (ㅁ). La ascitis es un trastorno cardiovascular que se observa predominantemente en pollos de crecimiento rápido más que en pollitos. En pollos de crecimiento rápido, la hipoxia hipobárica (falta de oxígeno) causa hipertrofia del ventrículo derecho (agrandamiento anormal) y expansión. Como resultado, el líquido se acumula en la región abdominal, lo que provoca la muerte por deficiencia de oxígeno ( $\underline{9})$. Existen dos hipótesis sobre el síndrome de ascitis: Hipótesis 1: En el desarrollo del síndrome de ascitis, las lesiones primarias se producen en los pulmones (hipertensión pulmonar) y, en consecuencia, el corazón se ve afectado (hipertrofia del ventrículo derecho). Hipótesis 2: La lesión primaria en el desarrollo del síndrome de la ascitis es causada por insuficiencia ventricular izquierda inducida por hipertensión sistémica que conduce a hipertrofia ventricular derecha y acidosis $(\underline{10})$. Se ha informado que la prevalencia de la ascitis ha aumentado en los últimos años. Las selecciones realizadas para lograr un crecimiento más rápido de los pollos han mejorado el potencial genético, pero también han causado algunas limitaciones anatómicas y fisiológicas, y la misma mejora ha tenido un impacto negativo en la salud de los animales. Un posible deterioro de los recursos de oxígeno (altitud, ventilación) estimulará el desarrollo de muchos mecanismos en el sistema cardiopulmonar, lo que desencadenará el síndrome de ascitis.

Se considera que la administración de suplementos de L-carnitina está relacionada con el síndrome de ascitis debido a su efecto sobre el metabolismo energético. Este estudio pretendió investigar los efectos de la L-carnitina como un medio potencial para reducir la incidencia de ascitis en pollos de engorde provocada por la altitud y su relación con parámetros fisiológicos y bioquímicos.

\section{MATERIAL Y MÉTODOS}

Animales, piensos y alimentación. En el ensayo se utilizaron 300 pollos de engorde machos (Ross 308) de un día de edad con similar peso corporal. En el ensayo, los animales fueron separados en 5 grupos, cada uno de los cuales fue dividido en 3 subgrupos. El grupo sin suplemento de L-carnitina fue el control, y los grupos suplementados con 100, 150, 200 y 250 mg/l de L-carnitina en agua potable fueron los grupos de tratamiento. La L-carnitina se utilizó en su forma de polvo blanco y puro; se obtuvo de Vimar Food Agriculture and Livestock Industry Trade Inc. Turquía. La L-carnitina se añadió al agua potable diariamente. La prueba se llevó a cabo en noviembre de 2014 y enero de 2015 a una altitud de 1.727 metros en Van-Turquía.

En la tabla 1 se presentan los ingredientes y los resultados de los análisis de nutrientes de los piensos utilizados en el ensayo. Los pollos de engorde fueron alimentados con alimento inicial durante los días 1 a 10 , seguidos por alimento de los productores durante los días 10 a 21 , y alimento de los acabadores durante los días 21 a 35. Se formuló una dieta basal de harina de maíz y soja de acuerdo con la NRC (1994). Los pollos de engorde recibieron alimento en pellets durante todo el período. Los alimentos y el agua se ofrecieron libremente a todos los grupos. El ensayo duró 35 días. Se aplicó un programa de iluminación de 24 horas durante todo el ensayo.

Bioquímica sanguínea, valores minerales, hormonales y de gases sanguíneos. Para los análisis bioquímicos de la sangre se tomaron muestras de sangre durante el sacrificio de 6 animales de cada subgrupo. Treinta minutos después de obtenidas las muestras de sangre, los sueros fueron separados por centrifugación durante 5 minutos a $4000 \mathrm{rpm}$ y se almacenaron en tubos ependorfs a $-20^{\circ} \mathrm{C}$ hasta su análisis. El análisis bioquímico de la sangre fue realizado por el dispositivo de recuento sanguíneo completo marca Swelab Alfa (Suecia) en muestras de sangre total. Todas las muestras fueron analizadas el mismo día. Las hormonas T3 y T4 se detectaron en el plasma con el autoanalizador de hormonas Elecsys e-411 de Roche Diagnostic (Estados Unidos) utilizando los kits de la marca Roche. En el día 35 del ensayo, se tomaron muestras de sangre de las venas alares de 3 ascitis y 3 pollos sanos de cada subgrupo en jeringas que contenían heparina para evitar la formación de coágulos. Los parámetros de gasometría, oximetría, electrolitos y metabolitos se midieron con el Radiómetro ABL-700 analizador de gas sanguíneo.

Estadísticas. Se empleó el análisis de varianza basado en el procedimiento del Modelo Lineal General (PROC GLM) de acuerdo con el diseño del ensayo (diseño experimental de parcelas aleatorias) y se utilizó la Prueba de Rango Múltiple de Duncan para comparar los promedios de los grupos. Los análisis estadísticos fueron realizados por el software del paquete estadístico SAS (versión 9.2).

\section{RESULTADOS}

Parámetros de rendimiento. En la tabla 2 se indican los pesos en vivo de los grupos de tratamiento semanalmente. Cuando se compararon los grupos en términos de aumento de peso vivo, las diferencias entre los promedios del grupo sólo a los $70,14^{\circ}$ y $28^{\circ}$ días resultaron ser estadísticamente significativas $(p<0.05)$. A los 35 días, al final del ensayo, no se registraron diferencias significativas entre los grupos con respecto al peso vivo $(p>0.05)$.

La suplementación de L-carnitina en agua no tuvo efectos significativos en el consumo acumulado de alimento de pollos de engorde por semanas y al final del ensayo 
Tabla 1. Composición $(\mathrm{g} / \mathrm{kg}$ ) y contenido de nutrientes (\%) de los piensos mezclados utilizados en el ensayo.

\begin{tabular}{|c|c|c|c|}
\hline Ingredientes & $\begin{array}{l}\text { Dieta de inicio para pollos de } \\
\text { engorde (Días 1-10) }\end{array}$ & $\begin{array}{l}\text { Dieta del productor de pollos de } \\
\text { engorde (Días 10-21) }\end{array}$ & $\begin{array}{l}\text { Dieta para terminar el pollo de } \\
\text { engorde (Días 21-35) }\end{array}$ \\
\hline Maíz & 411.07 & 450.70 & 460.74 \\
\hline Harina de soja (\%46HP) & 197.90 & 120.31 & 119.71 \\
\hline Soja entera & 140.00 & 160.00 & 160.00 \\
\hline Harina de gluten de maíz (\%60HP) & 86.75 & 64.26 & 20.00 \\
\hline Arroz (partido) & 40.00 & 40.00 & 40.00 \\
\hline Harina de pescado (\%53-60) & 40.00 & 40.00 & 60.00 \\
\hline Harina de girasol (\%36) & 30.00 & 40.00 & 40.00 \\
\hline DCP (\%18 P) & 10.44 & 5.73 & 2.69 \\
\hline Harina de pluma de pollo & 10.00 & 10.00 & 10.00 \\
\hline Harina de carne y hueso $(\% 35 \mathrm{HP})$ & 10.00 & 28.38 & 30.00 \\
\hline Extracto de maíz (graso) & - & 20.00 & 20.00 \\
\hline Aceite de soja & - & - & 19.45 \\
\hline Polvo de mármol & 9.43 & 6.83 & 5.73 \\
\hline L-Lisina & 4.12 & 4.20 & 2.99 \\
\hline Bicarbonato de sodio & 3.20 & 2.65 & 1.82 \\
\hline DL-metionina & 2.21 & 2.09 & 2.01 \\
\hline Sal & 1.40 & 1.21 & 1.60 \\
\hline Premezcla vitamínica1 & 1.00 & 1.00 & 1.00 \\
\hline Premezcla mineral2 & 1.00 & 1.00 & 1.00 \\
\hline L-Treonina & 0.88 & 1.04 & 0.66 \\
\hline Anticoccidiales & 0.60 & 0.60 & 0.60 \\
\hline Total & 1000.00 & 1000.00 & 1000.00 \\
\hline \multicolumn{4}{|c|}{ Nutrientes analizados $(\%)$} \\
\hline Materia seca & 89.44 & 89.19 & 89.26 \\
\hline Proteína bruta & 25.70 & 23.74 & 20.95 \\
\hline Grasa bruta & 4.42 & 5.12 & 7.65 \\
\hline Celulosa en bruto & 3.11 & 2.48 & 3.11 \\
\hline Ceniza bruta & 5.81 & 5.45 & 5.07 \\
\hline \multicolumn{4}{|c|}{ Nutrientes calculados (\%) } \\
\hline ME (kcal/kg) & 3020 & 3120 & 3220 \\
\hline Lisina & 1.44 & 1.34 & 1.21 \\
\hline Metionina & 0.68 & 0.64 & 0.57 \\
\hline Calcio & 1.05 & 0.95 & 0.85 \\
\hline Fósforo & 0.81 & 0.74 & 0.70 \\
\hline Sodio & 0.18 & 0.17 & 0.16 \\
\hline
\end{tabular}

$(p>0.05)$. Sin embargo, al final del ensayo, la mayor cantidad de consumo de alimento se observó en el grupo que recibió $100 \mathrm{mg} / \mathrm{l}$ de L-carnitina, mientras que la menor cantidad se observó en el grupo que recibió 150 $\mathrm{mg} / \mathrm{l}$ de L-carnitina.

Como se observa en la tabla 2, no se detectaron diferencias significativas en las tasas de conversión alimenticia entre grupos a lo largo del ensayo $(p>0.05)$. Sin embargo, en el grupo que recibió un suplemento de L-carnitina de $200 \mathrm{mg} / \mathrm{l}$, la relación de eficiencia alimenticia mejoró numéricamente (1.38).

Como se observa en la tabla 2 , no se registraron diferencias significativas en los valores de consumo de agua entre los grupos a lo largo del ensayo ( $p>0.05)$. Por otro lado, aunque no fue estadísticamente significativo, la ingesta total de agua fue mayor en el grupo suplementado con $100 \mathrm{mg} / \mathrm{I}$ de L-carnitina en comparación con los otros grupos al final del ensayo.

Tasas de mortalidad. Los efectos de la administración de suplementos de L-carnitina en las tasas de mortalidad de los pollos de engorde se presentan en la tabla 3. La tasa de mortalidad más baja $(18.33 \%)$ se registró en el grupo suplementado con $100 \mathrm{mg} / \mathrm{l}$ de L-carnitina, mientras que la tasa de mortalidad más alta (28.33\%) se registró en los grupos suplementados con 200 y 250 $\mathrm{mg} / \mathrm{l}$ de L-carnitina. 
Tabla 2. Los efectos de la suplementación con L-carnitina en el agua sobre los parámetros de rendimiento y los valores de consumo de agua $(\mathrm{ml})$ de los pollos de engorde.

\begin{tabular}{|c|c|c|c|c|c|c|c|}
\hline \multirow[b]{2}{*}{ DIAS } & \multicolumn{7}{|c|}{ GRUPOS DE TRATAMIENTO } \\
\hline & CONTROL & $\begin{array}{c}100 \mathrm{mg} / \mathrm{L} \\
\text { L-carnitina }\end{array}$ & $\begin{array}{c}150 \mathrm{mg} / \mathrm{L} \\
\text { L-carnitina }\end{array}$ & $\begin{array}{c}200 \mathrm{mg} / \mathrm{L} \\
\text { L-carnitina }\end{array}$ & $\begin{array}{c}250 \mathrm{mg} / \mathrm{L} \\
\text { L-carnitina }\end{array}$ & SEM & $\mathbf{p}=$ \\
\hline \multicolumn{8}{|c|}{ Peso en Vivo, $\mathbf{g}$} \\
\hline Día 0 & 46.61 & 46.53 & 46.34 & 46.63 & 46.59 & 0.235 & 0.995 \\
\hline Día 7 & $207.63^{\mathrm{ab}}$ & $210.84^{a}$ & $209.93^{a}$ & $200.51^{b}$ & $213.95^{a}$ & 1.396 & 0.005 \\
\hline Día 14 & $568.00^{\mathrm{a}}$ & $532.22^{\mathrm{b}}$ & $558.63^{a}$ & $519.54^{b}$ & $575.78^{a}$ & 3.443 & 0.001 \\
\hline Día 21 & 1095.84 & 1088.81 & 1087.84 & 1065.59 & 1090.39 & 7.033 & 0.699 \\
\hline Día 28 & $1809.95^{a}$ & $1808.51^{\mathrm{a}}$ & $1699.46^{b}$ & $1737.04^{\mathrm{ab}}$ & $1715.75^{\mathrm{ab}}$ & 14.975 & 0.042 \\
\hline Día 35 & 2475.56 & 2484.30 & 2486.94 & 2456.42 & 2386.84 & 23.470 & 0.689 \\
\hline \multicolumn{8}{|c|}{ Consumo de alimentos, $\mathbf{g}$} \\
\hline Día 7 & 211.25 & 213.05 & 211.15 & 206.75 & 211.93 & 1.027 & 0.239 \\
\hline Día 14 & 458.82 & 510.28 & 478.78 & 465.85 & 497.79 & 9.417 & 0.256 \\
\hline Día 21 & 789.93 & 785.33 & 753.93 & 803.07 & 797.24 & 11.357 & 0.525 \\
\hline Día 28 & 1045.90 & 1050.59 & 968.00 & 999.40 & 961.50 & 16.481 & 0.179 \\
\hline Día 35 & 1181.19 & 1184.35 & 1151.04 & 1140.43 & 1240.48 & 36.835 & 0.847 \\
\hline Total & 3687.08 & 3743.59 & 3562.95 & 3615.49 & 3708.93 & 44.302 & 0.541 \\
\hline \multicolumn{8}{|c|}{ Relación de eficiencia alimenticia, kg de consumo de alimento/kg de ganancia de peso vivo } \\
\hline Día 7 & 1.31 & 1.31 & 1.29 & 1.35 & 1.27 & 0.019 & 0.577 \\
\hline Día 14 & 1.27 & 1.59 & 1.37 & 1.47 & 1.38 & 0.041 & 0.116 \\
\hline Día 21 & 1.51 & 1.41 & 1.41 & 1.46 & 1.54 & 0.031 & 0.392 \\
\hline Día 28 & 1.45 & 1.45 & 1.48 & 1.51 & 1.48 & 0.040 & 0.978 \\
\hline Día 35 & 1.60 & 1.67 & 1.49 & 1.38 & 1.58 & 0.068 & 0.525 \\
\hline \multicolumn{8}{|c|}{ Consumo de agua $(\mathrm{ml})$} \\
\hline Día 7 & 390.33 & 381.33 & 377.33 & 372.10 & 377.67 & 5.388 & 0.754 \\
\hline Día 14 & 1050.33 & 1074.67 & 1006.33 & 1018.33 & 1036.67 & 25.279 & 0.845 \\
\hline Día 21 & 1703.27 & 1847.93 & 1736.27 & 1715.47 & 1638.03 & 58.234 & 0.762 \\
\hline Día 28 & 2751.00 & 2915.00 & 2844.63 & 2601.70 & 2500.20 & 87.828 & 0.393 \\
\hline Día 35 & 3571.07 & 3940.40 & 3721.73 & 3248.40 & 3150.87 & 113.085 & 0.107 \\
\hline Total & 9465.67 & 10159.33 & 9686.37 & 8956.03 & 8703.43 & 265.058 & 0.292 \\
\hline
\end{tabular}

*: Las diferencias entre los promedios de los grupos denotados por diferentes letras en la misma fila son estadísticamente significativas ( $<<0.05)$. SEM: Error estándar de diferencia entre medias.

Tabla 3. Los efectos de la suplementación con L-carnitina en las tasas de mortalidad por ascitis en pollos de engorde.

\begin{tabular}{ccc}
\hline $\begin{array}{c}\text { Grupos de } \\
\text { tratamiento }\end{array}$ & $\begin{array}{c}\text { Número de muertes } \\
\text { por ascitis }\end{array}$ & $\begin{array}{c}\text { Tasa de mortalidad } \\
\mathbf{( \% )}\end{array}$ \\
\hline Grupo de control & 12 & 20.00 \\
$100 \mathrm{mg} / \mathrm{l}$ L-carnitina & 11 & 18.33 \\
$150 \mathrm{mg} / \mathrm{l}$ L-carnitina & 16 & 26.67 \\
$200 \mathrm{mg} / \mathrm{l}$ L-carnitina & 17 & 28.33 \\
$250 \mathrm{mg} / \mathrm{l}$ L-carnitina & 17 & 28.33 \\
\hline
\end{tabular}

Plasma sanguíneo y parámetros del hemograma. Los efectos de la suplementación de L-carnitina en el agua sobre el plasma sanguíneo seleccionado y los parámetros del hemograma se muestran en la tabla 4. Al final del ensayo, la suplementación con L-carnitina no dio lugar a diferencias significativas entre los grupos en los parámetros del plasma sanguíneo aspartato aminotransferasa (AST), alanina aminotransferasa (ALT), fosfatasa alcalina (ALP), proteína total, colesterol, concentración de lipoproteínas de baja densidad (LDL), triyodotironina (T3) y parámetros del hemograma volumen corpuscular medio (MCV), ancho de distribución de glóbulos rojos (RDW), ancho de distribución real de glóbulos rojos (RDWa), concentraciones de hematocrito, plaquetas y hemoglobina $(p>0.05)$. Por otro lado, se registraron diferencias significativas en los valores de lipoproteínas de alta densidad (HDL), triglicéridos, creatina cinasa, glucosa, tiroxina (T4), recuento de glóbulos rojos (RBC), hemoglobina corpuscular media (HCM) y concentración media de hemoglobina corpuscular $(\mathrm{HCMC})$ entre los grupos $(p<0.05)$.

Parámetros de gases sanguíneos. Se midieron gases sanguíneos, oximetría, electrolitos y metabolitos en las muestras de sangre tomadas de 3 pollos con ascitis y 3 pollos sanos de cada subgrupo. Los efectos de la suplementación con L-carnitina sobre los parámetros de gases en sangre de los pollos de engorde ascíticos y sanos se presentan en la tabla 5 . La suplementación de L-carnitina en agua no tuvo efectos significativos sobre el hematocrito, sodio $(\mathrm{Na})$, potasio $(\mathrm{K})$, calcio $(\mathrm{Ca})$, bicarbonato de plasma $\left(\mathrm{HCO}_{3} \mathrm{p}\right)$, bicarbonato estándar $\left(\mathrm{HCO}_{3} \mathrm{pst}\right)$, exceso de base ( $\mathrm{C}$ Base $\left.\mathrm{B}\right)$, el gas sanguíneo, los electrolitos y los valores de metabolitos de pollos de engorde asciticos y sanos $(p>0.05)$. Por otro lado, los valores de Pouvoir hidrógeno $(\mathrm{pH})$, presión parcial de dióxido de carbono $\left(\mathrm{pCO}_{2}\right)$, presión parcial de oxígeno $\left(\mathrm{pO}_{2}\right)$, cloro $(\mathrm{Cl})$, hemoglobina $(\mathrm{Hb})$, concentración respiratoria de dióxido de carbono $\left(\mathrm{CtCO}_{2} \mathrm{~B}\right)$, presión parcial de concentración de dióxido de carbono ( $\left.\mathrm{CtCO}_{2} \mathrm{P}\right)$, anión $\mathrm{K}$, separación del anión, saturación de oxígeno 
Tabla 4. Los efectos de la suplementación con L-carnitina en el agua en el plasma sanguíneo seleccionado y los parámetros de hemografía de los pollos de engorde

\begin{tabular}{|c|c|c|c|c|c|c|c|}
\hline \multirow[b]{2}{*}{ Parámetros } & \multicolumn{7}{|c|}{ Grupos de tratamiento } \\
\hline & Control & $\begin{array}{l}100 \mathrm{mg} / \mathrm{L} \\
\text { L-carnitina }\end{array}$ & $\begin{array}{c}150 \mathrm{mg} / \mathrm{L} \\
\text { L-carnitina }\end{array}$ & $\begin{array}{l}200 \mathrm{mg} / \mathrm{L} \\
\text { L-carnitina }\end{array}$ & $\begin{array}{l}250 \mathrm{mg} / \mathrm{L} \\
\text { L-carnitina }\end{array}$ & SEM & $\mathbf{p}=$ \\
\hline \multicolumn{8}{|c|}{ Parámetros del plasma sanguíneo } \\
\hline AST ( U/L) & 292.68 & 238.90 & 275.28 & 290.03 & 249.06 & 12.788 & 0.456 \\
\hline ALT ( U/L) & 7.10 & 4.14 & 5.68 & 5.30 & 5.88 & 0.392 & 0.091 \\
\hline $\operatorname{ALP}(\mathrm{U} / \mathrm{L})$ & 2913.18 & 2875.25 & 3681.17 & 3012.12 & 2525.14 & 225.836 & 0.745 \\
\hline Proteína total (g/dl) & 3.35 & 3.10 & 2.98 & 3.01 & 3.03 & 0.061 & 0.242 \\
\hline Colesterol (mg/dl) & 133.50 & 119.36 & 108.67 & 111.56 & 114.13 & 3.029 & 0.065 \\
\hline $\mathrm{LDL}(\mathrm{mg} / \mathrm{dl})$ & 14.49 & 10.40 & 9.60 & 12.31 & 10.80 & 1.187 & 0.696 \\
\hline $\mathrm{HDL}(\mathrm{mg} / \mathrm{dl})$ & $118.15^{\mathrm{a}}$ & $95.10^{\mathrm{b}}$ & $92.68^{b}$ & $93.60^{b}$ & $91.34^{b}$ & 2.902 & 0.006 \\
\hline Triglicéridos (mg/dl) & $51.47^{\mathrm{ab}}$ & $37.14^{b}$ & $41.00^{b}$ & $34.33^{b}$ & $60.38^{a}$ & 2.616 & 0.012 \\
\hline Creatina quinasa (CK) (U/L) & $15744.58^{\mathrm{ab}}$ & $9893.33^{b}$ & $20395.20^{\mathrm{ab}}$ & $24622.83^{a}$ & $8605.25^{b}$ & 585.238 & 0.057 \\
\hline Glucosa (mg/dl) & $298.67^{a b}$ & $283.57^{b}$ & $286.50^{\mathrm{b}}$ & $285.44^{b}$ & $313.63^{a}$ & 3.208 & 0.021 \\
\hline $\mathrm{T} 3(\mathrm{pg} / \mathrm{ml})$ & 6.85 & 6.22 & 4.27 & 6.45 & 7.99 & 0.400 & 0.151 \\
\hline $\mathrm{T} 4(\mathrm{ng} / \mathrm{dl})$ & $0.86^{\mathrm{a}}$ & $0.86^{\mathrm{a}}$ & $0.91^{\mathrm{a}}$ & $0.66^{\mathrm{a}}$ & $0.40^{\mathrm{b}}$ & 0.041 & 0.001 \\
\hline \multicolumn{8}{|c|}{ Hemogram Parameters } \\
\hline RBC $\left(10^{12} / \mathrm{I}\right)$ & $2.98^{\mathrm{a}}$ & $2.83^{\mathrm{ab}}$ & $2.71^{\mathrm{b}}$ & $2.89^{a b}$ & $2.71^{\mathrm{b}}$ & 0.030 & 0.021 \\
\hline MCV (fl) & 134.55 & 135.38 & 134.02 & 136.82 & 139.60 & 0.636 & 0.163 \\
\hline RDW ( \%) & 9.79 & 9.65 & 9.30 & 9.76 & 10.15 & 0.118 & 0.438 \\
\hline RDWa (fl) & 77.55 & 77.39 & 74.22 & 79.16 & 82.65 & 1.041 & 0.339 \\
\hline Hematocrito (\%) & 40.19 & 38.34 & 36.30 & 39.64 & 37.78 & 0.475 & 0.083 \\
\hline Plaqueta (trombocito) $\left(10^{9} / \mathrm{l}\right)$ & 3.77 & 4.38 & 3.17 & 4.20 & 4.50 & 0.207 & 0.295 \\
\hline Hemoglobina $(g / d l)$ & 17.68 & 16.96 & 17.65 & 17.38 & 17.28 & 0.283 & 0.851 \\
\hline $\mathrm{MCH}(\mathrm{pg})$ & $59.22^{\mathrm{b}}$ & $59.89^{b}$ & $65.20^{\mathrm{a}}$ & $60.02^{b}$ & $63.85^{\mathrm{ab}}$ & 0.706 & 0.041 \\
\hline $\mathrm{MCHC}(\mathrm{g} / \mathrm{dl})$ & $43.97^{b}$ & $44.24^{b}$ & $48.70^{\mathrm{a}}$ & $43.84^{b}$ & $45.68^{\mathrm{ab}}$ & 0.496 & 0.025 \\
\hline
\end{tabular}

*: Las diferencias entre los promedios de los grupos denotados por diferentes letras en la misma columna son estadísticamente significativas ( $p<0.05$ ). SEM: Error estándar de diferencia entre medias

Tabla 5. Los efectos de la suplementación con L-carnitina en el agua sobre los parámetros de gases en sangre de pollos de engorde ascíticos y saludables.

\begin{tabular}{|c|c|c|c|c|c|c|c|c|}
\hline \multirow{2}{*}{\multicolumn{2}{|c|}{$\begin{array}{c}\text { Parámetros de gases } \\
\text { en sangre }\end{array}$}} & \multicolumn{7}{|c|}{ Grupos de tratamiento } \\
\hline & & Control & $\begin{array}{c}100 \mathrm{mg} / \mathrm{I} \\
\text { L-carnitina }\end{array}$ & $\begin{array}{c}150 \mathrm{mg} / \mathrm{I} \\
\text { L-carnitina }\end{array}$ & $\begin{array}{c}200 \mathrm{mg} / \mathrm{I} \\
\text { L-carnitina }\end{array}$ & $\begin{array}{c}250 \mathrm{mg} / \mathrm{I} \\
\text { L-carnitina }\end{array}$ & SEM & $\mathbf{p}=$ \\
\hline \multirow{4}{*}{$\mathrm{pH}$} & Saludable & $7.48^{\mathrm{A}}$ & 7.41 & $7.46^{\mathrm{A}}$ & $7.44^{A}$ & $7.37^{A}$ & 0.013 & 0.070 \\
\hline & Ascítico & $7.21 b^{B}$ & $7.42^{a}$ & $7.32^{\mathrm{abB}}$ & $7.32^{\mathrm{abB}}$ & $7.29^{\mathrm{bB}}$ & 0.016 & 0.032 \\
\hline & SEM & 0.034 & 0.030 & 0.017 & 0.021 & 0.041 & & \\
\hline & $\mathrm{P}$ & 0.009 & 0.837 & 0.008 & 0.036 & 0.361 & & \\
\hline \multirow{4}{*}{$\mathrm{pCO}_{2}$} & Saludable & $37.00^{\mathrm{B}}$ & 40.00 & $38.50^{\mathrm{B}}$ & $40.00^{\mathrm{B}}$ & $39.00^{\mathrm{B}}$ & 0.880 & 0.904 \\
\hline & Ascítico & $66.00^{\mathrm{A}}$ & 45.00 & $60.20^{A}$ & $61.86^{A}$ & $69.80^{\mathrm{A}}$ & 2.472 & 0.122 \\
\hline & SEM & 4.195 & 2.915 & 3.425 & 3.431 & 6.088 & & \\
\hline & $\mathrm{P}$ & 0.014 & 0.349 & 0.019 & 0.019 & 0.042 & & \\
\hline \multirow{4}{*}{$\mathrm{pO}_{2}$} & Saludable & $38.50^{\mathrm{A}}$ & 42.00 & 40.00 & $35.50^{A}$ & $43.50^{\mathrm{A}}$ & 2.199 & 0.488 \\
\hline & Ascítico & $6.00^{\mathrm{B}}$ & 27.50 & 13.80 & $4.43^{B}$ & $11.60^{\mathrm{B}}$ & 2.473 & 0.115 \\
\hline & SEM & 2.140 & 4.257 & 5.581 & 2.348 & 6.541 & & \\
\hline & $\mathrm{P}$ & 0.001 & 0.137 & 0.053 & 0.001 & 0.047 & & \\
\hline \multirow{4}{*}{ hematocrito } & Saludable & 31.00 & 31.00 & 40.00 & 31.50 & 29.00 & 2.604 & 0.406 \\
\hline & Ascítico & 41.67 & 34.00 & 45.80 & 43.57 & 39.00 & 3.215 & 0.946 \\
\hline & SEM & 2.273 & 12.083 & 1.706 & 3.130 & 8.804 & & \\
\hline & $\mathrm{P}$ & 0.102 & 0.651 & 0.128 & 0.111 & 0.570 & & \\
\hline \multirow{4}{*}{$\mathrm{Na}$} & Saludable & 154.00 & 150.50 & 152.00 & 151.00 & 150.00 & 0.810 & 0.419 \\
\hline & Ascítico & 144.40 & 151.50 & 143.80 & 143.00 & 148.80 & 1.332 & 0.268 \\
\hline & SEM & 1.788 & 1.118 & 2.699 & 2.740 & 1.480 & & \\
\hline & $P$ & 0.070 & 0.591 & 0.156 & 0.211 & 0.682 & & \\
\hline
\end{tabular}




\begin{tabular}{|c|c|c|c|c|c|c|c|c|}
\hline \multirow{4}{*}{$\mathrm{K}$} & Saludable & 6.75 & 4.85 & 5.10 & 5.15 & 5.50 & 0.531 & 0.549 \\
\hline & Ascítico & 8.20 & 3.95 & 7.02 & 7.29 & 6.50 & 0.399 & 0.098 \\
\hline & SEM & 0.779 & 0.471 & 0.864 & 0.705 & 0.446 & & \\
\hline & $P$ & 0.365 & 0.309 & 0.288 & 0.196 & 0.284 & & \\
\hline \multirow{4}{*}{$\mathrm{Ca}$} & Saludable & 1.30 & 1.23 & 1.37 & 1.37 & 1.26 & 0.038 & 0.440 \\
\hline & Ascítico & 1.25 & 1.18 & 1.22 & 1.26 & 1.18 & 0.041 & 0.933 \\
\hline & SEM & 0.059 & 0.049 & 0.101 & 0.057 & 0.077 & & \\
\hline & $P$ & 0.669 & 0.547 & 0.491 & 0.390 & 0.597 & & \\
\hline \multirow{4}{*}{$\mathrm{Cl}$} & Saludable & $109.00^{\mathrm{A}}$ & $109.50^{\mathrm{A}}$ & 110.50 & 109.00 & 109.50 & 1.349 & 0.984 \\
\hline & Ascítico & $100.80^{\mathrm{B}}$ & $107.00^{\mathrm{B}}$ & 100.60 & 99.86 & 103.60 & 1.172 & 0.416 \\
\hline & SEM & 1.658 & 0.353 & 2.414 & 2.419 & 1.260 & & \\
\hline & $\mathrm{P}$ & 0.045 & 0.037 & 0.079 & 0.118 & 0.054 & & \\
\hline \multirow{4}{*}{$\mathrm{Hb}$} & Saludable & 10.00 & 11.00 & 13.00 & 10.20 & $9.20^{B}$ & 0.842 & 0.369 \\
\hline & Ascítico & 13.53 & 14.00 & 14.94 & 14.19 & $15.93^{A}$ & 0.683 & 0.783 \\
\hline & SEM & 0.762 & 4.005 & 0.127 & 1.040 & 0.457 & & \\
\hline & $P$ & 0.105 & 0.649 & 0.568 & 0.114 & 0.001 & & \\
\hline \multirow{4}{*}{$\mathrm{HCO} 3 \mathrm{p}$} & Saludable & 27.10 & 24.45 & 27.00 & 26.75 & 23.35 & 1.316 & 0.633 \\
\hline & Ascítico & 25.52 & 28.40 & 30.12 & 31.10 & 31.88 & 0.811 & 0.073 \\
\hline & SEM & 1.532 & 1.645 & 1.085 & 1.451 & 1.774 & & \\
\hline & $\mathrm{P}$ & 0.605 & 0.231 & 0.185 & 0.200 & 0.050 & & \\
\hline \multirow{4}{*}{$\mathrm{HCO} 3$ pst } & Saludable & 24.20 & 24.20 & 27.05 & 26.50 & 23.00 & 0.756 & 0.199 \\
\hline & Ascítico & 21.43 & 26.55 & 24.88 & 25.36 & 26.20 & 0.728 & 0.260 \\
\hline & SEM & 2.184 & 1.151 & 0.768 & 1.161 & 1.579 & & \\
\hline & $P$ & 0.519 & 0.285 & 0.191 & 0.656 & 0.307 & & \\
\hline \multirow{4}{*}{ C BaseB } & Saludable & 0.10 & 0.50 & 3.50 & 2.90 & -3.10 & 1.176 & 0.248 \\
\hline & Ascítico & -1.85 & 3.65 & 3.45 & 3.50 & 4.20 & 0.972 & 0.412 \\
\hline & SEM & 5.444 & 3.040 & 0.800 & 1.335 & 2.453 & & \\
\hline & $\mathrm{p}$ & 0.818 & 0.553 & 0.973 & 0.838 & 0.222 & & \\
\hline \multirow{4}{*}{$\mathrm{ctCO} 2 \mathrm{~B}$} & Saludable & 21.50 & $22.5^{\mathrm{B}}$ & 23.95 & 24.75 & 22.00 & 0.863 & 0.483 \\
\hline & Ascítico & 24.63 & $25.10^{\mathrm{A}}$ & 27.48 & 28.70 & 29.60 & 0.795 & 0.231 \\
\hline & SEM & 2.017 & 0.145 & 1.037 & 1.156 & 2.014 & & \\
\hline & $\mathrm{P}$ & 0.442 & 0.006 & 0.128 & 0.151 & 0.094 & & \\
\hline \multirow{4}{*}{$\mathrm{ctCO} 2 \mathrm{P}$} & Saludable & 28.20 & 25.65 & 28.20 & 28.00 & $24.35^{\mathrm{B}}$ & 1.329 & 0.616 \\
\hline & Ascítico & 27.56 & 29.75 & 31.96 & 33.01 & $34.02^{\mathrm{A}}$ & 0.840 & 0.079 \\
\hline & SEM & 1.540 & 1.691 & 1.151 & 1.510 & 1.827 & & \\
\hline & $P$ & 0.833 & 0.228 & 0.141 & 0.161 & 0.036 & & \\
\hline \multirow{4}{*}{ cCa } & Saludable & 1.35 & 1.23 & 1.41 & 1.40 & 1.24 & 0.037 & 0.208 \\
\hline & Ascítico & 1.20 & 1.18 & 1.17 & 1.20 & 1.16 & 0.020 & 0.991 \\
\hline & SEM & 0.072 & 0.035 & 0.091 & 0.046 & 0.072 & & \\
\hline & $\mathrm{P}$ & 0.271 & 0.422 & 0.223 & 0.086 & 0.571 & & \\
\hline \multirow{4}{*}{ Anión K } & Saludable & 22.80 & 21.40 & 19.60 & 20.40 & 22.90 & 0.961 & 0.533 \\
\hline & Ascítico & $26.28^{a}$ & $20.05^{b}$ & $20.10^{\mathrm{b}}$ & $19.33^{b}$ & $19.82^{\mathrm{b}}$ & 0.689 & 0.007 \\
\hline & SEM & 2.137 & 0.506 & 0.698 & 0.988 & 1.463 & & \\
\hline & $P$ & 0.498 & 0.200 & 0.717 & 0.625 & 0.311 & & \\
\hline \multirow{4}{*}{$\begin{array}{l}\text { Brecha de } \\
\text { Anión }\end{array}$} & Saludable & 17.70 & 16.55 & 14.50 & 15.25 & 18.30 & 0.649 & 0.172 \\
\hline & Ascítico & $18.08^{a}$ & $16.10^{\mathrm{ab}}$ & $13.08^{a b}$ & $12.04^{b}$ & $13.32^{\mathrm{ab}}$ & 0.755 & 0.050 \\
\hline & SEM & 1.403 & 0.617 & 1.314 & 1.366 & 1.340 & & \\
\hline & $\mathrm{P}$ & 0.907 & 0.657 & 0.588 & 0.305 & 0.103 & & \\
\hline \multirow{4}{*}{$\mathrm{SO} 2$} & Saludable & $76.95^{A}$ & 76.10 & $77.85^{\mathrm{A}}$ & $70.10^{\mathrm{A}}$ & $78.20^{\mathrm{A}}$ & 3.091 & 0.764 \\
\hline & Ascítico & $4.90^{\mathrm{bB}}$ & $52.00^{\mathrm{a}}$ & $21.10^{\mathrm{bB}}$ & $5.26^{\mathrm{bB}}$ & $18.98^{\mathrm{bB}}$ & 4.106 & 0.034 \\
\hline & SEM & 2.316 & 6.692 & 9.784 & 3.958 & 10.682 & & \\
\hline & $\mathrm{P}$ & $<.001$ & 0.125 & 0.026 & 0.001 & 0.031 & & \\
\hline \multirow{4}{*}{ ctO2 } & Saludable & $10.80^{A}$ & 11.50 & $14.20^{\mathrm{A}}$ & $10.05^{\mathrm{A}}$ & 10.35 & 0.779 & 0.225 \\
\hline & Ascítico & $1.47^{\mathrm{B}}$ & 10.40 & $4.14^{\mathrm{B}}$ & $0.84^{\mathrm{B}}$ & 5.28 & 1.003 & 0.070 \\
\hline & SEM & 0.889 & 3.080 & 1.888 & 0.598 & 2.736 & & \\
\hline & $P$ & 0.023 & 0.824 & 0.035 & 0.000 & 0.344 & & \\
\hline
\end{tabular}

*: Las diferencias entre los promedios de grupo indicados por letras pequeñas diferentes en la misma fila son estadísticamente significativas ( $<<0.05$ ). $* *$ : Las diferencias entre los promedios de grupo denotados con letras mayúsculas diferentes en la misma columna son estadísticamente significativas ( $\mathrm{p}<0.05$ ). SEM: Error estándar de diferencia entre medias. 
$\left(\mathrm{SO}_{2}\right)$ y contenido de oxígeno $\left(\mathrm{ctO}_{2}\right)$ se vieron afectados de forma significativa por los tratamientos $(p<0.05)$. El valor del $\mathrm{pH}$ de la sangre disminuyó significativamente en los pollos ascíticos en comparación con los sanos.

\section{DICUSIÓN}

Similar a nuestros hallazgos de peso vivo, se ha reportado que la suplementación de $100 \mathrm{mg} / \mathrm{kg}$ de L-carnitina en las raciones de pollos de engorde con diferentes niveles de energía (11); $900 \mathrm{mg} / \mathrm{kg}$ de suplementación de L-carnitina en el $5 \%$ de la grasa animal que contiene las raciones de pollos de engorde (4); y 400, 600 y $800 \mathrm{mg} / \mathrm{kg}$ de suplementación de L-carnitina líquida y de potencia en las raciones de pollos de engorde (12), que el aditivo de L-carnitina no tiene ningún efecto significativo sobre el aumento de peso vivo al final del ensayo $(p<0.05)$. Contrario a la investigación actual, hay estudios que reportan que la L-carnitina contribuye significativamente al aumento de peso vivo. Parsaeimehr y et al (13) informaron de que la adición de $300 \mathrm{mg} / \mathrm{kg}$ de L-carnitina a las raciones que contenían diferentes niveles de grasa animal mejoró significativamente el aumento de peso vivo. Jalali et al (14) reportaron aumentos significativos en el peso vivo al final de la prueba de 35 días de adición de L-carnitina $(120 \mathrm{mg} /$ $\mathrm{kg}$ ) a raciones que contenían aceite de soja y aceite de girasol. Las diferencias observadas entre estos hallazgos de la investigación pueden atribuirse a las diferencias en las raciones, los niveles de dosis utilizados y las combinaciones de otros aditivos utilizados junto con los suplementos de L-carnitina. En resumen, el hecho de que no haya diferencias estadísticas entre los grupos, tal como revelan los datos de peso en vivo obtenidos al final del ensayo, indica que las dosis de los suplementos de L-carnitina utilizados no están en las concentraciones que cambiarán el valor del peso en vivo positiva o negativamente.

Murali et al (4) y Corduk et al (11) informaron que la suplementación con L-carnitina en las raciones de pollos de engorde no tuvo un efecto significativo en el consumo de alimento. Los resultados de estos investigadores están acordes con nuestros hallazgos que indican que la adición de diferentes niveles de L-carnitina en el agua potable no afecta significativamente el consumo de alimento de los pollos de engorde. Por otro lado, también hay estudios que demuestran que el suplemento de L-carnitina afecta significativamente el consumo de alimento. Wang et al (15) informaron que el suplemento de L-carnitina (100 $\mathrm{mg} / \mathrm{kg}$ ) redujo significativamente el consumo de alimento en pollos de engorde criados a bajas temperaturas ambientales. Tales diferencias entre los resultados de las investigaciones pueden atribuirse a los niveles de dosis del suplemento de L-carnitina utilizado, los niveles de energía de la ración y las especies animales utilizadas. El hecho de que no haya diferencias estadísticas entre los grupos referidos a partir de los valores de ingesta de alimento obtenidos al final del estudio indica que los niveles de dosis de L-carnitina utilizados no tienen un efecto significativo sobre el apetito en los pollos de engorde.

Murali et al (4); Taklimi et al (12) y Wang et al ( $\underline{15})$ reportaron que el suplemento de L-carnitina no tuvo un efecto significativo en la eficiencia alimenticia de los pollos de engorde. Asimismo, Geng et al (16) realizaron un estudio para determinar los efectos de los aditivos de L-carnitina y coenzima Q10 sobre el sistema inmunológico y el rendimiento de crecimiento de los pollos contra la ascitis. Se utilizó L-carnitina a dosis de 0,75 y $100 \mathrm{mg} / \mathrm{kg}$ y coenzima $\mathrm{Q} 10$ a dosis de 0 y $40 \mathrm{mg} / \mathrm{kg}$, respectivamente. Como resultado de la prueba, el ratio de eficiencia alimenticia en el grupo suplementado sólo con L-carnitina mejoró numéricamente. Michalczuk et al (17) informaron que la L-carnitina complementada con agua potable ( $62.5 \mathrm{~g} / 100$ litros) mejora numéricamente la eficiencia alimenticia. Los hallazgos de estos investigadores están de acuerdo con los hallazgos de este estudio. Esta coherencia puede atribuirse al hecho de que los niveles de aditivos de L-carnitina utilizados no afectan al consumo de piensos. Contrariamente a estos hallazgos, los estudios han demostrado que la L-carnitina contribuye significativamente a la relación de eficiencia alimenticia. Sharifi et al (18) encontraron que el suplemento de L-carnitina $(100 \mathrm{mg} / \mathrm{kg})$ con proteína reducida $(3 \%)$ mejoró significativamente la eficiencia alimenticia. Las diferencias observadas entre los resultados de estas investigaciones pueden atribuirse a las diferencias en las especies animales y en la ración utilizada.

La ingesta total de agua fue numéricamente mayor en el grupo suplementado con $100 \mathrm{mg} / \mathrm{L}$-carnitina en comparación con los otros grupos al final del ensayo. Esto puede atribuirse al hecho de que el grupo que recibió un suplemento de L-carnitina de 100 mg/l consumió una mayor cantidad de alimento en comparación con los otros grupos. Aunque no se encontró ninguna diferencia estadísticamente significativa entre los grupos en términos de consumo de agua, se observó que el consumo de agua disminuyó numéricamente con el aumento de los niveles de suplementación de L-carnitina. Esta conclusión sugiere que los pollos de engorde serán de gran importancia para determinar los requisitos de L-carnitina.

En el estudio actual se encontró que la tasa de mortalidad en el grupo que recibió un suplemento de L-carnitina de $100 \mathrm{mg} / \mathrm{l}$ era menor que la del grupo de control. El aumento de las tasas de mortalidad por ascitis en la quinta semana y hacia el final del ensayo puede atribuirse al aumento de la demanda de oxígeno debido al aumento del peso vivo de los animales y a la incapacidad de satisfacerlos adecuadamente, así como al uso de alimento en forma de gránulos. Zohair et al (19) reportaron que en comparación con el alimento en polvo, el alimento en gránulos desencadena ascitis en pollos de engorde criados a grandes altitudes. La tasa de mortalidad por ascitis en el estudio actual es menor. Esto puede atribuirse al hecho de que los animales no fueron sometidos a estrés por frío, lo que desencadena la ascitis. En contraste con el presente estudio, la tasa de mortalidad por ascitis fue de $3.75 \%$ para el grupo criado en condiciones normales de temperatura; $12.5 \%$ para el grupo de estrés por frío y $8.75 \%$ para el grupo expuesto al estrés por frío y que recibió L-carnitina $(100 \mathrm{mg} / \mathrm{kg})$ en el el estudio de Tan et al (20). Estas tasas de mortalidad por ascitis son más bajas que las obtenidas en el ensayo actual.

Çördük et al (11) informaron que la suplementación con L-carnitina en las raciones de pollos de engorde no afectó significativamente los niveles de glucosa en sangre, colesterol y triglicéridos. Asimismo, los niveles de colesterol total, LDL y triglicéridos ( $\underline{21})$; los niveles de hemoglobina y MCV (22); los niveles de colesterol y 
LDL en suero ( $\underline{23})$; los niveles de glucosa en sangre, LDL, colesterol, triglicéridos y proteína total $(\underline{13})$ y el valor de hematocrito en suero (18) no se vieron afectados de manera significativa por el aditivo de L-carnitina en las raciones de pollos de engorde. Estos resultados concuerdan con las conclusiones del presente estudio.

Contrariamente a los hallazgos existentes, Çördük et al (11) reportaron un aumento significativo en los niveles de AST de pollos alimentados con raciones de alta energía. Şimşek et al (24) reportaron que los valores de WBC, $\mathrm{RBC}$, hemoglobina y hematocrito fueron mayores en ratas suplementadas con L-carnitina. Elgazzar et al ( $\underline{25})$ revelaron que la suplementación con L-carnitina redujo significativamente los niveles de colesterol, triglicéridos, LDL, AST, ALT y ALP en conejos de Nueva Zelanda. Jali et al (14) encontraron que el suplemento de L-carnitina en las raciones que contenían aceite de soja y aceite de girasol redujo significativamente los niveles de triglicéridos al tiempo que aumentaba significativamente los niveles de proteína total en la sangre, colesterol, HDL y LDL. Las diferencias entre estos resultados pueden atribuirse a las diferencias en los niveles de energía de las raciones utilizadas, las fuentes de energía y el material animal.

Geng et al (16), Yousefi et al (26) reportaron que el suplemento de L-carnitina redujo significativamente el valor del hematocrito en pollos de engorde. En el presente estudio, con el suplemento de L-carnitina, el valor del hematocrito en sangre tendió a disminuir numéricamente, aunque no estadísticamente. Las hormonas de la glándula tiroides $(T 3, T 4)$ están directamente relacionadas con el desarrollo de los animales, ya que aumentan el metabolismo basal. Cuando la glándula tiroides está funcionando mal, hay una desaceleración en el desarrollo de los animales debido al trastorno que ocurre en el metabolismo basal (27). En el presente estudio, los niveles plasmáticos de T4 se redujeron significativamente con altas dosis de suplemento de L-carnitina ( $250 \mathrm{mg} / \mathrm{L})$. Esto puede estar relacionado con el hecho de que el aumento de peso vivo también es el más bajo de este grupo.

Mientras que el valor de $\mathrm{pH}$ de la sangre fue de 7.21 en pollos de engorde ascíticos, y de 7.48 en pollos de engorde sanos. Se observó que una dosis baja de suplemento de L-carnitina ( $200 \mathrm{mg} / \mathrm{l})$ aumenta el valor del $\mathrm{pH}$ en la sangre en pollos de engorde ascíticos $(p<0.05)$. Como se esperaba, el valor de $\mathrm{pCO}_{2}$ se encontró más alto en animales ascíticos en comparación con animales sanos, mientras que el valor de $\mathrm{pO}_{2}$ se encontró más bajo. Sin embargo, estos valores no fueron resultado de efectos estadísticamente significativos de la administración de suplementos de L-carnitina. El valor de $\mathrm{pCO}_{2}$ más bajo para los animales sanos fue de 37.00 y el valor más alto para los animales ascíticos fue de 69.80. El valor máximo de $\mathrm{pO}_{2}$ para los animales sanos fue de 43.50 y el valor mínimo para los animales ascíticos fue de 4,43 . Van As et al ( $\underline{28})$ reportaron que mientras que los niveles de $\mathrm{pCO}_{2}$ aumentaron significativamente en la ascitis, los niveles de $\mathrm{pO}_{2}$ se redujeron significativamente. Hafshejani et al (29) reportaron una disminución significativa en el valor del $\mathrm{pH}$ en animales ascíticos. Los hallazgos de estos investigadores son consistentes con los hallazgos del presente estudio. Aunque el valor de concentración de cloro no fue significativo para todos los grupos entre todos los animales sanos y ascíticos, fue estadísticamente significativo en el grupo de control y en el grupo que recibió $100 \mathrm{mg} / \mathrm{l}$ de suplemento de L-carnitina. No hubo diferencias estadísticas entre los otros grupos de tratamiento que puedan atribuirse a la gran varianza intragrupal. Las concentraciones de $\mathrm{SO}_{2}$ y $\mathrm{CtO}_{2}$ fueron mayores en animales sanos, mientras que las concentraciones de ctCO2P y hemoglobina fueron mayores en animales ascíticos $(p<0.05)$. El valor del hematocrito sanguíneo también aumentó numéricamente en animales ascíticos. Estos resultados concuerdan con las conclusiones de Tekeli ( $\underline{30})$. En pollos de engorde a gran altitud, el $\mathrm{SO}_{2}$ y el $\mathrm{FO}_{2} \mathrm{Hb}$ disminuyen la saturación de oxígeno en la sangre y aumentan los valores de cTHb y Hctc (hematocrito), lo que conduce a la ascitis.

En conclusión, el valor del pH de la sangre disminuyó significativamente en los pollos de engorde ascíticos en comparación con los pollos de engorde sanos. Se encontró que la administración de suplementos de L-carnitina es beneficiosa para disminuir las tasas de mortalidad por ascitis (hipertensión pulmonar). Estos efectos positivos pueden lograrse utilizando dosis bajas de suplementos de L-carnitina. Se espera que la administración de suplementos de L-carnitina en el agua de pollos de engorde alimentados con dietas de bajo contenido energético y proteínico pueda ser más eficaz para reducir las tasas de mortalidad por ascitis. Se necesita más investigación para confirmar estos beneficios.

\section{Conflictos de interés}

Los autores informan no tener ningún conflicto de interés.

\section{Agradecimientos}

Los autores agradecen a Van Yuzuncu Yıl Universidad (Proyecto BAP No. 2014 ZF-B179) por su apoyo financiero y técnico.

\section{REFERENCIAS}

1. Fathi E, Farahzadi R. Application of L-carnitine as nutritional supplement in veterinary medicine. Rom J Biochem. 2014; 51(1):31-41. URL Available in: http://journal.biochim.ro/archive/n51-1/pdfs 51-1/ rjb51-1 03.pdf

2. Harmeyer J. The physiological role of L-carnitine. Lohmann Information. 2002; 27:1-8. URL Available in: http://lohmann-information.de/content/l i 27 article 3.pdf
3. Flanagan $\mathrm{JL}$, Simmons PA, Vehige J, Willcox MDP, Garrett Q. Role of carnitine in disease. Nutr Metab. 2010; 7(30):1-14. DOI: https://doi. org/10.1186/1743-7075-7-30

4. Murali P, George SK, Ally K, Dipu MT. Effect of L-carnitine supplementation on growth performance, nutrient utilization and nitrogen balance of broilers fed with animal fat. Vet World. 2015; 8:482-486. DOI: https://doi.org/10.14202/ vetworld.2015.482-486 PMid:27047119 
5. DiNicolantonio JJ, Lavie $\mathrm{CJ}$, Fares $\mathrm{H}$, Menezes AR, O'Keefe JH. L-carnitine in the secondary prevention of cardiovascular disease: systematic review and meta-analysis. Mayo Clin Proc 2013; 88(6):544-551. DOI: https://doi.org/10.1016/j. mayocp.2013.02.007 PMid:23597877

6. Hurot JM, Cucherat M, Haugh M, Fouque D. Effects of L-carnitine supplementation in maintenance hemodialysis patients: A systematic review. J Am Soc Nephrol. 2002; 13:708-714. PMid:11856775

7. Zhou X, Liu F, Zhai S. Effect of L-carnitine and/or L-acetyl-carnitine in nutrition treatment for male infertility: A systematic review. Asia Pac J Clin Nutr. 2007; 16(1):383-390. PMid: $\underline{17392136}$

8. Singh PK, Shekhar P, Kumar K. Nutritional and management control of ascites sydrome in poultry. Int J of Livest Prod. 2011; 2(8):117-123. URL Available in: https://academicjournals.org/journal/ $\underline{\text { IJLP/article-abstract/DCC48CD1739 }}$

9. Baghbanzadeh A, Decuypere E. Ascites syndrome in broilers: physiological and nutritional perspectives. Avian Path. 2008; 37(2):117-126. DOI: https://doi. org/10.1080/03079450801902062 PMid: 18393088

10. Olkowski AA, Classen HL, Kumor L. Left atrioventricular valve degeneration, left ventricular dilation and right ventricular failure: A possible association with pulmonary hypertansion and aetiology of ascites in broiler chickens. Avian Pathol. 1998; 27(1):51-59. DOI: https://doi. org/10.1080/03079459808419274 PMid: 18484268

11. Corduk M, Ceylan N. Ildiz F. Effects of dietary energy density and L-carnitine supplementation on growth performance, carcass traits and blood parameters of broiler chickens. S Afr J Anim Sci. 2007; 37(2):6573. DOI: https://doi.org/10.4314/sajas.v37i2.4029

12. Taklimi SMM, Ghazvinian K, Kasgari MRA. Effect of L-carnitine on performance and carcass quality of broiler chickens. Acad J of Sci Res. 2015; 3(3):5054. URL Available in: https://academiapublishing. org/journals/ajsr/abstract/2015/Mar/Taklimi\%20 et\%20al.htm

13. Parsaeimehr K, Afrouziyeh M, Hoseinzadeh S. The effects of L-carnitine and different levels of anial fat on performance, carcass characteristics, some blodd parameters and immune response in broiler chicks. Iran j Appl Anim Sci. 2014; 4(3):561566. URL Available in: http://ijas.iaurasht.ac.ir/ article 513564.html

14. Jalali SMA, Rabiei R. Kheiri F. Effects of dietary soybean and sunflower oils with and without L-carnitine supplementation on growth performance and blood biochemical parameters of broiler chicks. Arch Anim Breed. 2015; 58:387-394. DOI: https:// doi.org/10.5194/aab-58-387-2015
15. Wang $Y W$, Ning $D$, Peng $Y Z$, Guo YM. Effects of dietary L-carnitine supplementation on growth performance, organ weight, biochemical Parameters and Ascites Susceptibility in Broilers Reared Under Low-temperature Environment. Asian-Australas ] Anim Sci. 2013; 26(2):233-240. DOI: https://doi. org/10.5713/ajas.2012.12407 PMid:25049781

16. Geng A, Li B, Guo Y. Effects of dietary L-carnitine and coenzyme Q10 at different supplemental ages on growth performance and some Immune response in ascites-susceptible broilers. Arch Anim Nutri. 2007; 61(1):50-60. DOI: https://doi. org/10.1080/17450390601117041 PMid: $\underline{17361948}$

17. Michalczuk M, Lukasiewicz M, Niemiec J, Wnuk A, Mroczek-Sosnowska N. Effetc of L-carnitine on performance and dressing percentage of broiler chickens. Ann Warsaw Univ of Life Sc SGGW Anim Sci. 2012; 51:89-99. URL Available in: http:// annals-wuls.sggw.pl/?q=node/550

18. Sharifi MR, Hassanpour H, Khajali F. Dietary L-carnitine aupplement counteracts pulmonary hypertensive response in broiler chickens fed reduced-protein diets and subjected to cool condition and hypobaric hypoxia. J Poultry Sci. 2015; 52:206-212. DOI: https://doi.org/10.2141/ jpsa.0140205

19. Zohair GAM, Al-Maktari GA, Amer MM. A comparative effect of mash and pellet feed on broiler perfromance and ascites at high altitude (Field study). Glob Vet. 2012; 9(2):154-159. URL Available in: https://www. idosi.org/gv/GV9(2)12/8.pdf

20. Tan X, Hu S, Wang L. The effect of dietary L-carnitine supplementation on pulmonary hypertension syndrome mortality in broilers exposed to low temperatures. J Anim Phys Anim Nutri. 2008; 92:203-210. https://doi.org/10.1111/j.14390396.2007.00727.x PMid: 18336417

21. Hossininezhad MM, Irani M, Seidavi A. Dietary effects of L-carnitine supplement on performance and blood parameters of broiler chickens. J Food Agr Environ. 2011; 9(3-4):475-481. URL Available in: https:// www.wflpublisher.com/Abstract/2307

22. Asadi $H$, Sadeghi AA, Eila N, Aminafshar $M$. The effects of ractopamine, coenzyme $Q$ and L-carnitine supplementation, individual or in combination, on the hematological parameters of broiler chickens. World Appl Sci J. 2013; 21(1):69-72. URL Available in: https://pdfs.semanticscholar.org/a3ae/ afadcbdc1a5a6b40feadd74946b85148274f.pdf

23. Murali P, George SK, Merey AD, Dipu MT, Narayanankutty K. Effects of L-carnitine supplementation on serum lipid profile of broiler chicken fed with animal fat. Inter J Agri \& Food Sci Techn. 2013; 4(8):773-774. URL Available in: https://www.ripublication.com/ijafst_spl/ ijafstv4n8spl 09.pdf 
24. Şimşek N, Karadeniz A, Bayraktaroğlu AG. Ratlarda periferal kan hücreleri üzerine L-karnitin, arı Sütü ve nar çekireğinin etkileri. Kafkas Univ Vet Fak Derg. 2009; 15(1):63-69. DOI: https://doi.org/10.9775/ kvfd.2008.68-A

25. Elgazzar UB, Ismael I, Ghanema A, Kalaba ZM. Effect of dietary L-carnitine supplementation on the concentration of circulating serum metabolites in growing New Zealand rabbits. Aust J Basic \& Appl Sci. 2012; 6(2):80-84. URL Available in: http://www. ajbasweb.com/old/ajbas/2012/February/80-84.pdf

26. Yousefi A, Khajali F, Hassanpour H, Khajali Z. Dietary L-carnitine improves pulmonary hypertensive response in broiler chickens subjected to hypobaric hypoxia. J Poultry Sci. 2013; 50:143-149. https:// doi.org/10.2141/jpsa.0120145

27. Albay R, Şen MK, Temelli S. Broiler üretiminde suların dezenfeksiyonunda iyot kullanımının karkas özellikleri üzerine etkisi. Van Vet J. 2002; 13(12):20-24. URL Available in: http://vfdergi.yyu.edu. tr/archive/2002/13_1-2/2002_13_(1-2)_20-24.pdf
28. Van As $P$, Elferink MG, Closter $A M$, Vereijken $A$, Bovenhuis $\mathrm{H}$, Crooijmans RPMA, Decuypere $\mathrm{E}$, Groenen MAM. The Use of Blood Gas Parameters to Predict Ascites Susceptibility in Juvenile Broilers. Poult Sci. 2010; 89:1684-1691. https://doi. org/10.3382/ps.2009-00430 PMid:20634524

29. Hafshejani EF, Gholami-Ahangaran M, Hosseni E. Study of blood cells, blood gases and thyroid hormones in broiler chickens suspected of ascites Syndrome. Glob Vet. 2012; 8(1):18-21. URL Available in: https://idosi.org/gv/GV8(1)12/4.pdf

30. Tekeli A. Effects of ascites (Pulmonary hypertension) sydrome on blood gas, blood oximetrety parameters and heart sections of broilers grown at high altitude. J Anim Plant Sci. 2014; 24(4): 998-1002. URL Available in: http://www.thejaps.org.pk/ docs/v-24-4/04.pdf 